Bucknell University

Bucknell Digital Commons

\title{
Modern 'Junk Food' and Minimally-processed 'Natural Food' Cafeteria Diets Alter the Response to Sweet Taste but Do Not Impair Flavor-Nutrient Learning in Rats
}

\author{
Kristen M. Palframan \\ Bucknell University \\ Kevin P. Myers \\ Bucknell University, kmyers@bucknell.edu
}

Follow this and additional works at: https://digitalcommons.bucknell.edu/fac_journ

\section{Recommended Citation \\ Palframan, Kristen M. and Myers, Kevin P.. "Modern 'Junk Food' and Minimally-processed 'Natural Food' Cafeteria Diets Alter the Response to Sweet Taste but Do Not Impair Flavor-Nutrient Learning in Rats." Physiology \& Behavior (2016) : 146-157.}

This Article is brought to you for free and open access by the Faculty Scholarship at Bucknell Digital Commons. It has been accepted for inclusion in Faculty Journal Articles by an authorized administrator of Bucknell Digital Commons. For more information, please contact dcadmin@bucknell.edu. 


\title{
Modern 'junk food' and minimally-processed 'natural food' cafeteria diets alter the response to sweet taste but do not impair flavor-nutrient learning in rats
}

\author{
Kristen M. Palframan ${ }^{\mathrm{b}}$, Kevin P. Myers ${ }^{\text {a,b,c,* }}$ \\ a Department of Psychology, Bucknell University, Lewisburg, PA, USA \\ b Animal Behavior Program, Bucknell University, Lewisburg, PA, USA

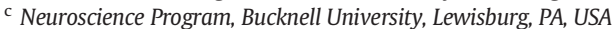

\section{H I G H L I G H T S}

- Flavor-nutrient learning is robust in animal experiments but less reliable in humans.

- This may reflect impairment by flavor-nutrient inconsistency in the modern diet.

- We studied rats fed highly-processed junk food vs. minimally-processed natural diets.

- We find no support for the 'flavor confusion' hypothesis.

- Both diets altered rats' responses to sweetness.

\section{A R T I C L E I N F O}

\section{Article history:}

Received 27 October 2015

Received in revised form 2 January 2016

Accepted 11 January 2016

Available online 12 January 2016

\section{Keywords:}

Food preference

Learning

Overeating

Food reward

Palatability

Lick microstructure

Progressive ratio

\begin{abstract}
A B S T R A C T
Animals learn to prefer and increase consumption of flavors paired with postingestive nutrient sensing. Analogous effects have been difficult to observe in human studies. One possibility is experience with the modern, processed diet impairs learning. Food processing manipulates flavor, texture, sweetness, and nutrition, obscuring ordinary correspondences between sensory cues and postingestive consequences. Over time, a diet of these processed 'junk' foods may impair flavor-nutrient learning. This 'flavor-confusion' hypothesis was tested by providing rats long-term exposure to cafeteria diets of unusual breadth ( 2 or 3 foods per day, 96 different foods over 3 months, plus ad libitum chow). One group was fed processed foods (PF) with added sugars/fats and manipulated flavors, to mimic the sensory-nutrient properties of the modern processed diet. Another group was fed only 'natural' foods (NF) meaning minimally-processed foods without manipulated flavors or added sugars/ fats (e.g., fresh fruits, vegetables, whole grains) ostensibly preserving the ordinary correspondence between flavors and nutrition. A CON group was fed chow only. In subsequent tests of flavor-nutrient learning, PF and NF rats consistently acquired strong preferences for novel nutrient-paired flavors and PF rats exhibited enhanced learned acceptance, contradicting the 'flavor-confusion' hypothesis. An unexpected finding was PF and NF diets both caused lasting reduction in ad lib sweet solution intake. Groups did not differ in reinforcing value of sugar in a progressive ratio task. In lick microstructure analysis the NF group paradoxically showed increased sucrose palatability relative to PF and CON, suggesting the diets have different effects on sweet taste evaluation.
\end{abstract}

(c) 2016 Elsevier Inc. All rights reserved.

\section{Introduction}

Food preferences and meal size are strongly determined by learning. Choices about which foods to select and how much of each food to consume are influenced by appearance, taste, odor, and texture. Evaluation of those sensory features is shaped by experience, especially by learning

\footnotetext{
* Corresponding author at: Department of Psychology, O'Leary Center, Bucknell University, Lewisburg, PA 17837, USA

E-mail address: kmyers@bucknell.edu (K.P. Myers).
}

to associate particular sensory cues with rewarding or aversive postingestive consequences that follow (see reviews [1-4]).

An extensive body of research with animals (primarily rats and mice [1,5,6], but across a range of mammalian species e.g., [7-9]) shows that one category of associative learning - flavor-nutrient conditioning powerfully influences preferences and intake. When a specific flavor is followed by nutrients sensed in the gastrointestinal tract or postabsorptively, animals shift their evaluation and respond to that flavor differently on subsequent encounters. These learned flavornutrient associations typically produce increased preference (choice of that flavor relative to others) and increased acceptance (larger amounts 
consumed) [10-12]. Effects are especially strong when the nutrient is glucose or a glucose-containing polysaccharide, but have also been shown with proteins and fats [6,13-16]. This is regarded as a type of Pavlovian conditioning in which the flavor is a conditioned stimulus (CS) associated with the unconditioned stimulus (US) effects of postingestive nutrient sensing.

In animal studies flavor-nutrient learning is rapid and robust, leading to the view that it is a potentially obesogenic influence in modern environments, as it promotes choice and intake of more energy dense foods. But it has been difficult to evaluate the putative involvement of flavor-nutrient learning in human overeating, since these effects have been unreliable in human studies (see review and discussion in [17, 18]). This poses a substantial dilemma for translational research: why is it that robust flavor-nutrient learning is easily demonstrated in animal studies but harder to obtain with human participants? Several possible reasons have been proposed and the present study is chiefly concerned with one: the notion that the modern, highly varied, processed diet interferes with or disrupts learning about flavor cues.

Modern food processing radically changes the sensory-nutrient relationships that would ordinarily exist in an unmanipulated omnivorous diet, by adding flavors and flavor enhancers, caloric and non-caloric sweeteners and fat substitutes, and physical manipulations of odor, texture, mouthfeel, and appearance. Processing manipulates macronutrient content and energy density, especially adding fats and highly refined carbohydrates that alter foods' physiological impacts. Many familiar foods also come in 'diet' or reduced-calorie versions engineered to taste as similar as possible to the higher energy versions. Thus consumers routinely encounter similar flavors in foods that differ substantially in energy content, and other foods that are nutritionally identical but differ only in their flavor. This experience may eventually cause flavor cues to be treated as uninformative and ignored by the relevant attentional and memory mechanisms. We call this the "flavor confusion" hypothesis.

This explanation seems plausible given the tremendous variability of foods in the modern environment. One recent study [19] observed that consumers in a major UK metropolitan area have ready access to 71 distinct brands and varieties of pepperoni pizza that span a three-fold variation in energy content, and that prior experience with more varieties was associated with reduced expected satiety and poorer compensatory adjustment of intake after a pizza preload. While that study provides evidence that experiential variability may impair adaptive responses in other encounters with that type of food, it does not address whether such experience impairs de novo learning about new foods or flavors. That study was also chiefly concerned with expected and experienced satiety, whereas flavor-nutrient learning in animal models is typically expressed in increased intake, not satiety.

Animal studies on the effects of inconsistent sensory-nutrient relationships have mainly focused on sweetness, as incorporating non-nutritive sweeteners is said to create a situation where sweetness inconsistently predicts postingestive carbohydrate loads. Several experiments have observed weight gain and impaired satiation responses to sweet foods in rats fed non-nutritive sweeteners [20-23]. This illustrate a possible impact of modern dietary variability, but sweetness is likely a unique case, in that it is already instinctively endowed with hedonic valence prior to any learning experience, and sweetness is simultaneously a powerful sensory US in its own right as well as a putative CS for subsequent postingestive effects. Thus the sweetener research does not necessarily address possible impairments of learned responses to initially-arbitrary flavor cues, which is a vital type of experiencedependent plasticity in food evaluation.

Relatively little animal work has experimentally modeled dietary variety specifically in the context of flavor-nutrient conditioning, and experiments have used only a small number flavored chows differing in energy density [24] or a range of "supermarket" foods (e.g., cheese, bologna, yogurt, bread, etc.) [25] which do provide sensory diversity but may not mimic the sensory-nutrient "confusion" inherent in the modern diet. But other experiments have shown that a history of eating a varied, high energy diet history can impair learning systems relevant to food choice and meal patterning [26], albeit not flavor-nutrient associations specifically.

Thus the purpose of the present work was to directly address the proposal that extensive experience with a highly variable diet of processed 'junk' foods impairs the ability to learn new flavor-nutrient associations. A key feature of the present experimental design was to compare flavor-nutrient learning in a chow-only control group versus two groups maintained on different types of cafeteria diets.

One cafeteria diet group was fed (in addition to ad lib chow) a wide variety of processed foods (PF) which have manipulated flavors and added sugars and/or fats. Foods were selected in an attempt to create extensive sensory-nutrient inconsistency, such as particular flavors appearing in many different types of foods (e.g., several different cheese-flavored, cocoa-flavored, apple-cinnamon flavored foods, etc.) as well as many nutritionally identical foods differing only in flavor (i.e., different flavors of the same brand of crackers or cereal).

The flavor confusion hypothesis depends not on the number of foods per se, but rather on flavor-nutrient inconsistency across those foods that is to say variability rather than variety (after [19]). Therefore a comparison cafeteria diet group received only minimally-processed "natural" foods (NF) including fruits, vegetables, nuts, legumes, whole grains, and meats, to provide variety without the flavor-nutrient inconsistency attributable to technological manipulation of flavors and energy content, and better approximating the range of sensory-nutrient relationships typical in the environment that shaped evolution of these dietary learning systems in the brain. This NF group is also notable since cafeteria diet studies typically involve energy-dense processed foods, and we are unaware of any prior studies of the psychological effects of this alternative diet. Of course the PF and NF diets differ in other respects (average energy density, macronutrient proportions, etc.) but this experiment focused on sensory-nutrient variability. Any group differences caused by these diets could be pursued in research with more controlled diets.

After three months on the cafeteria diets or chow-only, rats were tested for their ability to learn about novel flavors in a flavor-nutrient conditioning protocol. Rats were trained with a novel flavor $(\mathrm{CS}+)$ in a glucose solution and a different flavor (CS-) in glucose + saccharin mixture matched to the $\mathrm{CS}+$ for initial hedonic value but providing much less energy. Then rats were tested for preference and acceptance of two flavors, both now given in the glucose + saccharin solution. Rats that learned the flavor-nutrient association would prefer the CS+ flavor that had previously marked the higher-energy solution over the CSflavor. The flavor-confusion hypothesis predicts that extensive PF diet exposure would have deleterious effects on flavor-nutrient learning. Inclusion of the NF cafeteria group along with a chow-only CON group was to determine effects of dietary variety per se versus the putative disruptive effects of the modern, processed diet.

\section{General methods}

All procedures were approved by the Bucknell University IACUC and were consistent with the Guide for the Care and Use of Laboratory Animals, 8th Ed.

\subsubsection{Subjects}

Subjects were 36 female Sprague-Dawley rats from six litters born in our colony to breeding stock obtained from Sage Research Models (Boyertown, PA). Littermates were assigned to experimental conditions described below at approximately 8 weeks old. Rats were pair-housed with a littermate of the same condition except when behavioral testing required individual housing. Housing was in $8 \times 16 \times 10.5^{\prime \prime}$ plastic tub cages with corn cob bedding, in a colony room maintained at 
approximately $21^{\circ} \mathrm{C}$ and $40 \%$ humidity, with a $12: 12$ h light:dark cycle (lights on at 0800).

\subsubsection{Experimental conditions $\mathcal{E}$ diets}

The three dietary conditions were processed foods (PF) cafeteria diet, natural foods (NF) cafeteria diet, or chow-only control (CON). Condition assignments were pseudo-random by litter and weight, with two rats from each litter assigned to each condition to create experimental groups matched for initial body weight. CON rats received a standard cereal-based rodent chow ad libitum (Mazuri Laboratory Rodent Diet $5663,3.41 \mathrm{kcal} / \mathrm{g}, 14 \%$ energy from fat, $27 \%$ from protein, 59\% from carbohydrate). Cafeteria groups received the same chow plus limited portions each day of two or three different foods from their cafeteria diet. Water was available ad libitum.

"Processed foods" (PF) in this experiment were an extensive variety of packaged 'junk' foods which contain added sugars and/or fats, and have been processed to enhance their sensory characteristics, especially with added flavors. Examples are pastries, flavored granolas, sugary breakfast cereals, flavored snack chips, and prepared foods like canned baked beans and ready-to-eat microwavable pasta. Fruit and vegetable products were included in forms that involved added sugars and/or fats and flavor manipulations (e.g., canned pie fillings, canned yam casserole, fried plantain chips). In contrast, the "natural foods" (NF) cafeteria diet comprised minimally-processed foods as close to their natural state as practical. Importantly, these were foods containing no added sugars or fats and no manipulated flavorings, including a wide variety of fruits, vegetables, nuts, and whole grains. Foods like rice and whole grains were typically par-cooked with a small amount of water. Both cafeteria diets included meats, but again for the PF group these has added fats/sugars/flavorings (e.g., breakfast sausages, canned corned beef, prepared meat snacks) and for the NF group they were chosen to involve as little processing as practical and sanitary, containing simply the cooked meat itself without seasonings or added fats (for example, plain boiled eggs, boiled chicken breast, boiled pork liver). In both PF and NF diets all foods were solids and semi-solids (purees); none were liquids or beverages.

A changing selection of cafeteria foods was provided each afternoon in pre-weighed portions, in plastic dishes hung inside of the cage. Leftovers were recorded the next day, though for most foods rats consumed the entirety. Because the foods in the PF and NF diets differed so widely in composition, it would have been impossible to meaningfully match the PF and NF diets on a number of parameters. Since our main goal was to influence rats' experience with sensory variety, not to induce overeating or obesity, we provided relatively small, fixed "snack" amounts of the cafeteria foods daily in addition to ad lib chow, and matched the PF and NF groups only on the approximate weight of the foods given each day. This meant the average energy density of the PF diet exceeded the NF diet. Energy density of the average PF food was $3.85 \mathrm{kcal} / \mathrm{g}$ (range: $0.67-6.43 \mathrm{kcal} / \mathrm{g}$ ) and of NF was $1.66 \mathrm{kcal} / \mathrm{g}$ (range: $0.15-6.73 \mathrm{kcal} / \mathrm{g}$ ).

Initially, PF and NF groups were given two novel foods every day for 39 days ( 78 different foods). Then the foods were cycled through again in different combinations for another 39 days. Then rats received three foods per day (two familiar plus one novel) for the last 18 days. Typically PF rats received one or two sweet and one non-sweet (savory or salty) food every day whereas NF rats would typically receive one or two fruits/vegetables plus one legume, nut, grain, or meat. In total, each group was exposed to 96 different foods over the course of three months prior to the next phase of the study, with each individual food given between one and three separate occasions.

\section{Experiment 1A: flavor-nutrient preference learning}

The ability to learn a new flavor-nutrient relationship was assessed after the three months of cafeteria diet exposure. This procedure was adapted from Warwick \& Weingarten [27], who paired a CS+ flavor with $6.1 \%$ glucose and a CS- flavor with a mixture of $1 \%$ glucose $+0.125 \%$ saccharin, having found these two vehicles were equally preferred by naive rats. We first replicated this equivalence in a separate pilot experiment with 10 naive rats tested with a series of brief, twobottle tests across several days, between the $1 \%$ glucose $+0.125 \%$ saccharin mixture (hereafter " $G+S$ ") versus glucose concentrations from 1 to $8 \%$. We found $6.5 \%$ glucose to be equally preferred to $G+S$, a value quite close to the $6.1 \%$ used by Warwick \& Weingarten.

For the flavor-nutrient conditioning phase, cafeteria diets were discontinued and rats were trained with alternating exposures to one flavor (orange or lemon-lime) in $6.5 \%$ glucose and the opposite flavor in the equally-preferred but low energy $G+S$. Learned preference was then assessed in a two bottle choice between orange vs lemonlime, both mixed in $\mathrm{G}+\mathrm{S}$. The chief outcome of interest was whether either or both of the cafeteria diet groups differed from CON in this learned preference.

\subsubsection{Methods}

Rats were individually housed and fed only chow limited to the dark period. For two acclimation days rats received a bottle containing $20 \mathrm{~mL}$ of plain $\mathrm{G}+\mathrm{S}$ immediately after dark onset, prior to chow. Flavornutrient training was conducted over the next four days. Each day at lights out, rats received a single bottle containing $20 \mathrm{~mL}$ of either the $\mathrm{CS}+$ flavor in $6.5 \%$ glucose or the CS- flavor in $\mathrm{G}+\mathrm{S}$. The CS flavors were orange and lemon-lime (Kool-Aid, General Foods, Inc., $0.05 \mathrm{~g}$ unsweetened powder per $100 \mathrm{~g}$ of solution). These were selected because there had been no citrus flavors in the cafeteria diets, and because prior experiments in our lab have seen no inherent preference between them. Assignment of orange or lemon-lime as CS+ was counterbalanced. Intake was measured by weighing the bottles after $2 \mathrm{~h}$, then chow was provided and removed the next morning. Across days rats received two CS+ and two CS- sessions in alternating order.

Rats' preference for the CS + flavor vs CS- flavor was assessed in a two-bottle test repeated twice on consecutive days with the left-right positions reversed. Each rat was provided two bottles containing $40 \mathrm{~mL}$ of $\mathrm{G}+\mathrm{S}$ solution, one flavored with orange and the other with lemon-lime. Intake was measured after $2 \mathrm{~h}$, and the two session were averaged for analysis.

\subsubsection{Data analysis}

All analyses in this report were performed with IBM SPSS version 23.0. Bodyweights after the cafeteria feeding but prior to flavor-nutrient conditioning were compared in a one-way ANOVA, with pairwise contrasts between each group. Two-bottle preference tests following flavor-nutrient conditioning were analyzed with a 2 (Flavor) $\times 3$ (Group) mixed ANOVA, with flavor (CS+ vs CS-) as a withinsubjects factor and Group as a between-subjects factor. Post hoc tests determined whether each group preferred the CS+ over CS-, and whether the groups consumed similar or different amounts.

\subsubsection{Results}

By this stage in the experiment, diet history resulted in body weight differences (one-way ANOVA, main effect of Group, $\mathrm{F}(2,33)=9.44$, $p<0.01$ ). Average weights (mean \pm SD) for CON, PF, and NF were $286.1 \pm 17.2$, $339.3 \pm 47.5$, and $291.4 \pm 26.7$, respectively. Pairwise contrasts show that the PF rats were heavier than the CON, $t(22)=$ 3.94, $p<0.01$, and heavier than NF, $t(22)=3.44, p<0.01$, whereas CON and NF were similar to one another $(p=0.69)$.

All three groups acquired a strong CS+ flavor preference in flavornutrient conditioning. Intakes in the two-bottle choice between the $\mathrm{CS}+$ and CS- in $\mathrm{G}+\mathrm{S}$ are depicted in Fig. 1 and All groups consumed significantly more CS + than CS- (main effect of Flavor, $\mathrm{F}(1,33)=$ 
$192.8, p<0.001)$. Post hoc tests confirmed that CS + intake significantly exceeded CS- intake for each of the three groups individually (paired $t=4.93,10.18$, and 10.66 for PF, NF, and CON respectively, each $p<0.01, d f=11$ ) However there were some group differences. First, total intake varied across groups (main effect of Group on intake, $\mathrm{F}(2,33)=5.569, p<0.05)$. Repeated contrasts confirm CON rats' intake significantly exceeded both other groups. NF rats' intakes were significantly lower than CON and higher than PF. Second, the degree of difference between CS + vs CS- intake was not uniform across groups (Flavor $\times$ Group interaction, $\mathrm{F}(2,33)=7.97, p<0.05$ ). This interaction was explored with post hoc tests of simple main effects. CON consumed more CS + than either of the other groups (both $p<0.01$ ) which were similar to each other. CS- intake did not differ between the groups. Thus the significant interaction suggests CON more strongly preferred CS + over CS- than did PF and NF, which were similar to one another.

\subsubsection{Discussion}

The outcome of the first flavor-nutrient conditioning experiment was somewhat ambiguous. On the one hand, all three groups acquired a strong preference for the flavor paired with higher energy density. Thus at a basic level it can be immediately concluded that prior extensive exposure to the sensory complexity of the highly processed diet certainly did not prevent learning in the PF group. On the other hand, the learned preference appeared to be weaker among the cafeteria groups. Flavor-nutrient conditioning protocols typically produce quite robust preference shifts, with CS + preference sometimes near absolute, so even relatively small decreases in percent intake may represent a genuine attenuation. Yet some unexpected observations during the experiment make this finding equivocal.

First, we unexpectedly observed that during the initial familiarization exposures with $\mathrm{G}+\mathrm{S}$ and in the training sessions with flavored solutions, the CON rats consistently consumed more of the solutions than both cafeteria groups. This means CON rats accrued more experience with the flavors during training and also, on average, would have experienced more postingestive nutrient stimulation on $\mathrm{CS}+$ trials than the other groups. In other words, CON rats may have learned better because they actually provided themselves with more training. Second, the same tendency was seen in the two-bottle preference test, as CON rats again consumed significantly more overall than the other two groups. Thus

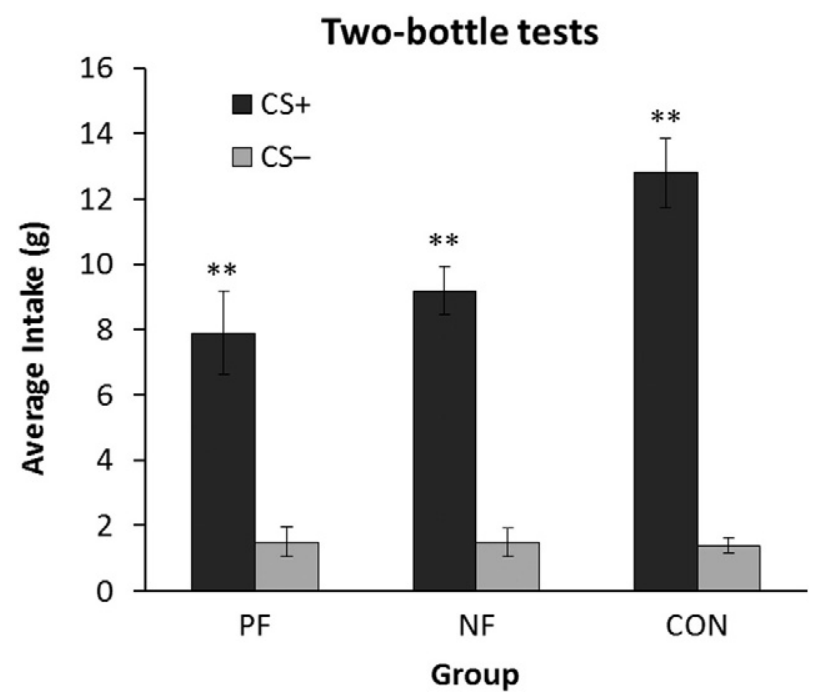

Fig. 1. Mean \pm SEM intakes by each group in the two-bottle preference test conducted after flavor-nutrient training in Experiment $1 \mathrm{~A}$. In this test the CS+ and CS- flavors (orange and lemon-lime) were both presented in the same $1 \%$ glucose $+0.125 \%$ saccharin vehicle solution, so preferential intake of the CS+ indicates flavor-nutrient learning. Asterisks indicate CS + intake is significantly greater than CS- intake within that group $\left({ }^{* *} p<0.01\right)$. it is ambiguous whether the appearance of stronger preference for $\mathrm{CS}+$ in that group genuinely represents a stronger learned stimulation of CS+ intake or is an artifact of their overall tendency to consume more, which was observable prior to training. These issues were addressed in Experiment 1B.

\section{Experiment 1B: flavor-nutrient conditioning of acceptance and preference}

To explore possible group differences in flavor-nutrient learning following PF, NF, or CON diet history, the same rats were studied in a second round of flavor-nutrient conditioning with some procedural differences aimed at resolving the ambiguities described above. First, intake during training sessions was limited to a smaller, fixed amount, to equate training exposure across groups. Second, instead of only measuring two-bottle preference for CS + vs CS- after training, we also measured rats' conditioned acceptance of the CS flavors in one-bottle tests. This was of interest because of the group differences that became apparent in ad lib intake of the sweet solutions. We sought to determine the extent to which CS + intakes differed between groups because of generalized group differences in sweet intake (which would be apparent in one-bottle acceptance of all three solutions) versus specific, learned differences in evaluation of the CS+ itself.

After flavor-nutrient training sessions, rats were tested in a series of one-bottle sessions for ad lib intake of unflavored $\mathrm{G}+\mathrm{S}$, the $\mathrm{CS}+$ flavor added to $\mathrm{G}+\mathrm{S}$, and the $\mathrm{CS}$ - flavor added to $\mathrm{G}+\mathrm{S}$, in order to determine if flavor-nutrient conditioning stimulated increased acceptance of the $\mathrm{CS}+$ flavor. That is, after having the opportunity to associate the CS+ flavor with caloric consequences, would addition of the CS+ flavor to the lower-calorie $G+S$ solution stimulate rats to consume more $\mathrm{G}+\mathrm{S}$ than what they'd typically consume of the unflavored $\mathrm{G}+\mathrm{S}$ itself? Two-bottle preference was also measured at the conclusion of the experiment, to determine if group differences in preference strength would replicate after equating each group's training intakes. As in Experiment $1 \mathrm{~A}$, the aim was to determine whether prior cafeteria diet experience impairs learning about a flavor paired with positive postingestive consequences.

\subsubsection{Methods}

All rats remained on chow only, restricted to the dark period. The CS flavors were coffee-flavored and butter-flavored extracts (McCormick brand, $0.4 \mathrm{~mL} / 100 \mathrm{~g}$ of solution). These flavor extracts were found in a separate pilot to be approximately equally preferred by naive rats, and no coffee- or butter-flavored foods were among the prior cafeteria diets.

For the first two days rats received a bottle of $40 \mathrm{~mL}$ of unflavored $\mathrm{G}+\mathrm{S}$ beginning immediately after lights out. Intake after $2 \mathrm{~h}$ was measured. For the next six days, one-bottle training sessions were conducted alternating across days between the CS+ flavor (coffee or butter) in $6.5 \%$ glucose, and the CS- flavor (the opposite, butter or coffee) in G $+\mathrm{S}$. Flavor/solution pairings were counterbalanced. In these sessions intake was limited to $12 \mathrm{~mL} / \mathrm{session}$, based on the intakes of the lowestconsuming rats in preceding unflavored $\mathrm{G}+\mathrm{S}$ sessions. This was done to equate training flavor experience across groups.

After training, one-bottle acceptance tests were conducted across three consecutive days. For these sessions, rats were provided a bottle containing $40 \mathrm{~mL}$ of solution for $2 \mathrm{~h}$ at the onset of the dark period, prior to chow. The stimulus order (unflavored $G+S$, $C S+$ in $G+S$, and $C S-$ in $G+S$ ) across days was counterbalanced within each experimental group. Bottles were removed and weighed after $2 \mathrm{~h}$ and chow was provided.

Finally, two-bottle tests for preference between coffee- and butterflavored $G+S$ were conducted as described in Experiment $1 \mathrm{~A}$. 


\subsubsection{Data analysis}

Intakes during training were analyzed with 2 (Flavor) $\times 3$ (Group) mixed ANOVA, with Flavor as the within-subjects factor and Group as a between-subjects factor, to determine if (as intended) limiting the volumes equated training intakes across the three groups. The series of one-bottle acceptance tests after training was analyzed with a 3 (Flavor) $\times 3$ (Group) mixed ANOVA, with flavor (unflavored G + S, CS + flavor in $\mathrm{G}+\mathrm{S}$, and $\mathrm{CS}-$ flavor in $\mathrm{G}+\mathrm{S}$ ) as a within-subjects factor. Planned contrasts then compared each groups' intake of CS + and of $\mathrm{CS}-$ to the unflavored $\mathrm{G}+\mathrm{S}$. A conditioned acceptance effect would be reflected in increased intake of $\mathrm{CS}+$ (but not CS-) relative to unflavored. Two-bottle preference data were analyzed as in Experiment $1 \mathrm{~A}$.

\subsubsection{Results}

As intended, limiting the training solutions to $12 \mathrm{~mL} /$ session equated the PF, NF, and CON rats' training intakes Though all three groups consumed somewhat more CS + than CS- across the course of training, $\mathrm{F}(1,33)=4.78, p<0.05$, intakes were uniform across the three groups (no main effect of Condition, $\mathrm{F}(2,33)=0.35$, n.s., nor Flavor $\times$ Condition interaction $\mathrm{F}(2,33)=1.29$, n.s. $)$.

\subsubsection{One-bottle acceptance tests}

The first outcome of interest was each group's one-bottle intakes of the $\mathrm{CS}+$ flavor in $\mathrm{G}+\mathrm{S}$, compared to unflavored $\mathrm{G}+\mathrm{S}$ and $\mathrm{CS}$ - flavor in $\mathrm{G}+\mathrm{S}$, to determine if flavor-nutrient learning specifically stimulated intake of the CS + flavor. Intakes are depicted in Fig. 2A. A 3 (Group) $\times 3$ (Flavor) repeated measures ANOVA on one-bottle intakes across groups showed a significant main effect of flavor, which confirms that in general rats responded differently to the three solutions $(F(2,66)=19.403$, $p<0.001)$. Within-subjects contrasts confirms that CS+ intake exceeded unflavored $\mathrm{G}+\mathrm{S}(\mathrm{F}(1,33)=34.81, p<0.001)$ whereas CSintake did not $(\mathrm{F}(1,33)=3.71$, n.s. $)$ Thus on average all rats learned to increase acceptance of the $\mathrm{CS}+$.

Total solution intake also significantly differed between groups (main effect of Group, $\mathrm{F}(2,33)=6.336, p<0.05)$. Planned contrasts reveal that CON rats had the largest overall intake, followed by PF then NF rats. The Group $\times$ Flavor interaction was not significant in this analysis $(\mathrm{F}(4,66)=0.831, p>0.05)$.

To further examine each group's pattern we conducted a repeated measures ANOVA on one-bottle intakes for each group separately, with planned contrasts to compare each CS with unflavored $G+S$. This analysis shows that CON rats acquired at best a weak acceptance of CS + and relatively little differential response to the flavors, since for CON there was no overall significant main effect of solution type on consumption $(F(2,22)=2.44$, n.s. $)$ and contrasts found that their nominally higher CS + intake was not significantly greater than their unflavored $\mathrm{G}+\mathrm{S}$ intake $(\mathrm{F}(1,11)=3.59, p=0.09)$. Quite differently from $\mathrm{CON}$, the two cafeteria groups showed strong increased acceptance of the CS + . The PF group consumed different amounts of the three stimuli (main effect of solution type $\mathrm{F}(2,22)=13.11, p<0.001$ ) with contrasts confirming they consumed more $C S+$ than $G+S$ $(\mathrm{F}(1,11)=19.72, p<0.001)$ but not more CS- than $\mathrm{G}+\mathrm{S}(\mathrm{F}(1,11)=$ 0.422 , n.s.). The NF group also treated the solutions differently (main effect $F(2,22)=10.45, p<0.01$ ) but contrasts suggest somewhat poorer differentiation between the two CSs. NF rats drank a significantly higher amount of $\mathrm{CS}+$ than $\mathrm{G}+\mathrm{S}[\mathrm{F}(1,11)=24.431, p<0.01]$ but also consumed more CS- than $\mathrm{G}+\mathrm{S}(\mathrm{F}(1,11)=6.205, p<0.05)$. In sum, CON rats treated the three solutions most similarly in the one-bottle tests, whereas PF rats showed strong, specific stimulation of CS+ intake. NF rats increased intake of both CSs but increased CS + acceptance more than CS-.

\subsubsection{Two-bottle preference tests}

All groups strongly preferred the CS+ flavor in the two-bottle tests. The intakes depicted in Fig. 2B were analyzed with a 2 (Flavor) $\times 3$ (Group) mixed ANOVA. Rats consumed significantly more CS + than CS- (main effect of flavor, $\mathrm{F}(1,33)=28.514, p<0.001$ ), and post hoc tests confirm this for each of the three groups individually (paired $t=5.32$, 3.16, and 3.01 for $\mathrm{PF}, \mathrm{NF}$, and CON respectively, each $p<0.01, d f=11$ ) Differences between the three groups that had been seen in Experiment $1 \mathrm{~A}$ were not replicated. Although CON rats appeared to have a slightly higher total intake (in this case seemingly due to higher CS- intake than the other groups) it was not a statistically significant effect (main effect of Group on total intake, $F(2,33)=1.51$, n.s.). Nor were there any differences in degree of CS+ preference (no Flavor $\times$ Group interaction, $F(2,33)=0.712$, n.s. $)$. Similar CS + preference strength across groups was confirmed by a one-way ANOVA comparing the three groups' percentage $\mathrm{CS}+$ intake $(\mathrm{CS}+$ intake / total intake $* 100)$, which found no significant group effect $(F(2,33)=2.28$, n.s.).

\subsubsection{Discussion}

The results of Experiment 1B extend and clarify those of 1A, first by showing again that both cafeteria diet groups learned to prefer the flavor cue paired with the postingestive effects of glucose over the comparison flavor matched for initial palatability but paired with lower
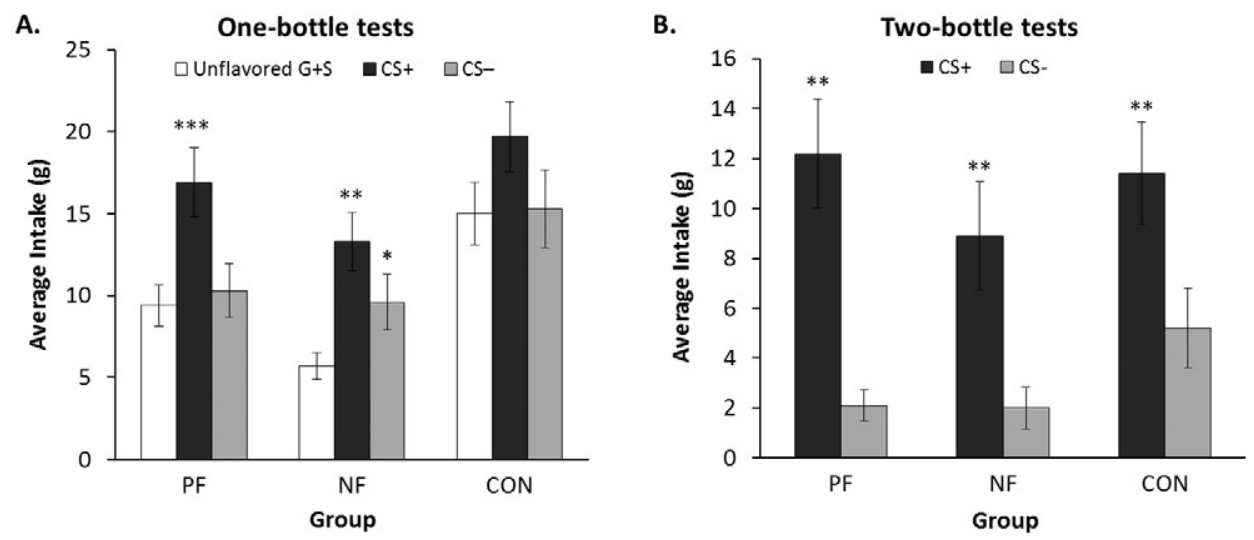

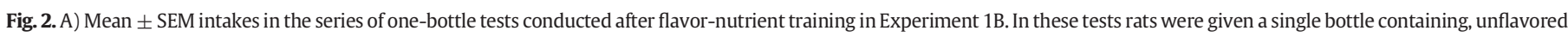

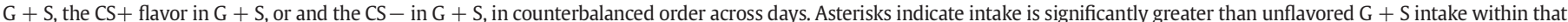

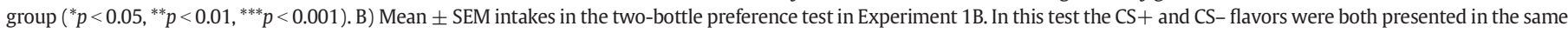

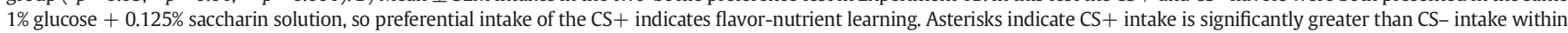
that group $\left({ }^{* *} p<0.01\right)$. 
energy density. There was no between-group difference in learned CS+ preference. Thus the stronger preference by CON rats seen in Experiment $1 \mathrm{~A}$ was likely an artifact. Thus there was no evidence for any impairment of flavor-nutrient preference learning following either type of cafeteria diet exposure.

Tests of one-bottle acceptance, which demonstrate the stimulation of meal size when a flavor cue has become associated with post-oral glucose sensing, revealed important effects of prior diet history which also contradict the flavor confusion hypothesis. First, both cafeteria diet groups showed stronger conditioned stimulation of CS + intake than the CON group, which showed a statistically negligible effect. Second, the PF group, which had the prior history of sensory complexity via a 'junk food' diet abundant in manipulated flavors, sugars, and fats and ostensibly poor flavor-nutrient correspondence, performed the best in this measure, showing substantial increased acceptance that was specific to the CS+. It is unlikely this apparent difference is secondary to reduced neophobia in the cafeteria groups, since in relevant tests in this and the prior experiment, $\mathrm{CON}$ rats consumed more than the other two groups, not less.

In these tests CON rats once again consumed more of the sweet solutions, suggesting cafeteria diet history attenuated some aspect of the attraction to sweet tastes. CON rats had higher initial G + S consumption in the acclimation sessions, and higher intakes of all solutions in the one-bottle tests than PF and NF. Conceivably, the relatively high baseline acceptance of $\mathrm{G}+\mathrm{S}$ by $\mathrm{CON}$ rats in this experiment could create a ceiling effect preventing expression of learned acceptance. These rats undoubtedly learned about the postingestive consequences paired with $\mathrm{CS}+$, as seen in their significant two-bottle preference, replicating prior experiments using a similar training protocol in ordinary rats [27]. Lack of increased CS + acceptance in CON is not a problematic outcome since increased acceptance may require more experience to emerge than the preference seen in two-bottle tests. In fact significant preference is evident after single-trial training [28]. Additional training would likely have produced a clearer CS + acceptance effect in the $\mathrm{CON}$ rats. But this underscores that cafeteria groups - especially PF learned readily, since the training significantly stimulated thier intake. Thus prior dietary diversity seems to have increased their sensitivity to this aspect of flavor-nutrient conditioning.

In sum, Experiments $1 \mathrm{~A}$ and $1 \mathrm{~B}$ not only cast doubt on the hypothesis that the modern, highly processed diet impairs flavor-nutrient learning, they show some evidence for the opposite. Since, by some measures the PF group showed superior learning, these results suggest the PF rats developed a sort of flavor expertise. Indeed the "confusing" nature of their cafeteria diet experience could be viewed as extensive practice learning about subtle differences in similarly-flavored foods. This idea is considered further in the General discussion.

\section{Experiment 2}

While Experiments $1 \mathrm{~A}$ and $1 \mathrm{~B}$ focused on effects of prior dietary experience on flavor-nutrient learning, they also revealed an unexpected effect of cafeteria diets on sweet consumption. In several test sessions in which rats consumed sweet solutions ad libitum, PF and NF rats typically consumed less than CON. This similarity between PF and NF was counterintuitive since they had quite different prior experiences with sweetness. PF rats had exposure to many highly sweetened, highenergy dense foods (pastries, cookies, candy) and many foods high in refined carbohydrates, whereas NF rats' experienced relatively low levels of simple sugars in fruits, and were routinely consuming unrefined, complex starches in vegetables, legumes, and whole grains. Yet both groups consistently consumed less sweet solutions than the CON rats, whose only prior sweetener experience was in the behavioral testing itself. It is possible that dietary variety per se could play a role in attenuating response to sweetness, or that repeated prior experience with any amount of sweetness reduces overall sweetness evaluation. But given their different histories it is likely that PF and NF rats were consuming less sweets than CON for different reasons.

Experiment 2 continued to study the same rats, first to confirm the lower sweet solution consumption by NF and PF rats compared to CON, and second, to investigate two behavioral measures which could help characterize the motivational or sensory-hedonic mechanisms underlying this group difference. Operant responding for sucrose reinforcers on a progressive ratio schedule was used to assess rats' motivation for sucrose reward, and lick microstructure analysis of sucrose consumption was used to assess hedonic evaluation.

In Experiment 2 we first measured each group's intake of a sweet, highly-preferred sucrose + saccharin mixture. The $6.5 \%$ glucose and $1 \%$ glucose $+0.125 \%$ saccharin mixture used in Experiments $1 \mathrm{~A}$ and $1 \mathrm{~B}$ are both sweet and acceptable to rats, yet could be considered relatively low-moderate among the entire range of sweetness intensity. This experiment used a sweeter mixture of $2 \%$ sucrose $+0.2 \%$ saccharin. We have previously found this solution to be avidly consumed because it is sweet but less satiating than high carbohydrate concentrations. Our first goal was determine if the group difference replicated with this sweeter, more highly preferred stimulus.

Next, rats were trained in an operant task to lever-press for sucrose reinforcers. Once trained, operant lever-pressing was observed on a progressive ratio reinforcement schedule, which means that as each pellet is earned, the number of lever presses required to earn the next pellet is progressively increased. Each rats' breakpoint - the point at which the rat ceases responding because the reinforcer is no longer sufficient to maintain the required workload - is taken as a measure of how motivated a rat is to obtain the sucrose reinforcer (e.g., [29-31]). Prior work has shown, for example, that PR breakpoint predicts individual differences in rats' ad lib sugar consumption [32].

Finally, we performed microstructural analysis of rats' licking patterns across a range of sucrose concentrations. Lick microstructure analysis is a method to infer the perceived hedonic value of taste solutions. As rats consume the solution an electronic lick sensor interfaced to a computer records each lick with millisecond resolution. This reveals a temporal pattern characterized by sustained periods of licking at a relatively constant rate (lick bursts) separated by occasional pauses of varying length. Extensive prior work (reviewed in [33-35]) has shown that specifically the average size of lick bursts - in other words, the degree to which rats exhibit sustained bursts of high-rate licking without pausing - indicates the subjective hedonic value of the tastant.

\subsection{Methods}

\subsubsection{Ad lib sweet consumption}

This stage began immediately after completion of Experiment 1B, and cafeteria diets were still withheld. All rats received chow limited to the dark phase of the light:dark cycle, though drinking water was available ad libitum. After 6 days of acclimation to this chow restriction, rats were tested at mid-day with $2 \mathrm{~h}$ access to a solution of $2 \% \mathrm{su}-$ crose $+0.2 \%$ saccharin. The same test was repeated the following day, and the two-session average was calculated.

\subsubsection{Progressive ratio lever-pressing for sucrose reward}

For this phase rats were fed restricted daily chow rations to maintain them at $90-95 \%$ of their initial weight in order to facilitate operant conditioning. Rats were weighed every $2-3$ days and their chow portions were individually adjusted accordingly to keep weights within the targeted range.

Operant training was conducted in four identical operant chambers equipped with a house light, a retractable lever, and a pellet dispenser that delivered $45 \mathrm{mg}$ sucrose pellets into an illuminable trough flanked by a head entry detector. Each chamber was inside a sound-attenuating cubicle. The house light in the ceiling of the chamber remained illuminated during the session. 
Rats were trained with one 30-min session per day. In initial shaping sessions, lever-insertions occurred on a 60-90 second ITI and were temporally paired with sucrose pellet delivery to auto-shape rats to approach and investigate the lever. Thereafter lever pressing was reinforced on a CRF schedule until rats attained a session-end criterion of 50 pellets. They were then transitioned to a FR-3 schedule and then an FR-5 schedule for one session each. For the final sessions rats were reinforced on a progressive ratio (PR) schedule, which requires the rat to press the lever a progressively increasing number of times to earn each successive pellet, following the formula [ $\left.\left(5 \times \mathrm{e}^{0.2 \mathrm{n}}\right)-5\right]$, as recommended by Richardson and Roberts [36]. The breakpoint, i.e., the point at which the response requirement has increased to where the reinforcer no longer maintains lever pressing, was considered reached when 15 min elapsed without a pellet earned. Each rat was tested in the PR three times on consecutive days, and the average of those three PR breakpoints was used.

\subsubsection{Lick microstructure}

Following the conclusion of the operant testing described in the previous section, cafeteria diets were resumed for three weeks. Then, at the outset of the next phase cafeteria diets were discontinued and rats were fed daily chow rations to maintain $95 \%$ of their new free-feeding weight, to maintain motivation to consume the sweet solutions in the series of daily lick microstructure sessions.

After several days on the restricted chow schedule, rats were familiarized with consuming sweet solutions in the lickometry chambers, an array of 10 identical cylindrical enclosures $(35 \mathrm{~cm}$ tall $\times 25 \mathrm{~cm}$ diameter) made of opaque plastic with a stainless steel wire grid floor. A narrow opening on the front wall enables the rat to access the drinking spout of a bottle held on a computer-controlled bottle retractor. The sipper tube is connected to an electronic lick sensor interfaced to the computer which records the timing of each lick for subsequent offline analysis. In two familiarization sessions rats received 30-min access to the $\mathrm{G}+\mathrm{S}$ solution that had been used in Experiment 1 .

Rats were tested with $5 \%, 10 \%$ and $30 \%$ (w/w) sucrose concentrations in a series of $30 \mathrm{~min} /$ day sessions. Order of concentration across days was counterbalanced across the first three sessions, then repeated in the opposite order across the next three sessions. Software converted the licking data binned into bursts of licking (defined as a period of sustained licking without a pause $>1 \mathrm{~s}$ ), to determine each rat's total licks, average burst size, and number of lick bursts for each session. Measures were averaged for the two tests at each sucrose concentration.

\subsubsection{Data analysis}

For ad libitum sweet consumption, progressive ratio breakpoints, and bodyweights, each of these dependent measures was analyzed in a one-way ANOVA across groups, with post hoc pairwise contrasts comparing the individual groups when justified by a significant main effect. For the lick microstructure tests, each dependent measure (licks per burst, number of bursts, and total licks) was analyzed with a 3 (Sucrose Concentration) $\times 3$ (Group) mixed ANOVA with sucrose concentration as a within-subjects factor.

\subsection{Results}

\subsubsection{Ad lib sweet consumption}

The goal of this phase was to verify our observations in Experiments $1 \mathrm{~A}$ and $1 \mathrm{~B}$ that both cafeteria diet groups consistently consumed less sweet solution than $\mathrm{CON}$ when given ad lib access. That finding was replicated, as seen in Fig. $3(F(2,33)=6.314, p<0.01)$. Planned contrasts confirm that the CON group drank more than the NF group $(t(33)=$ $3.31, p<0.01)$ and the PF group $(t(33)=2.78, p<0.01)$, with PF and $\mathrm{NF}$ similar to one another.

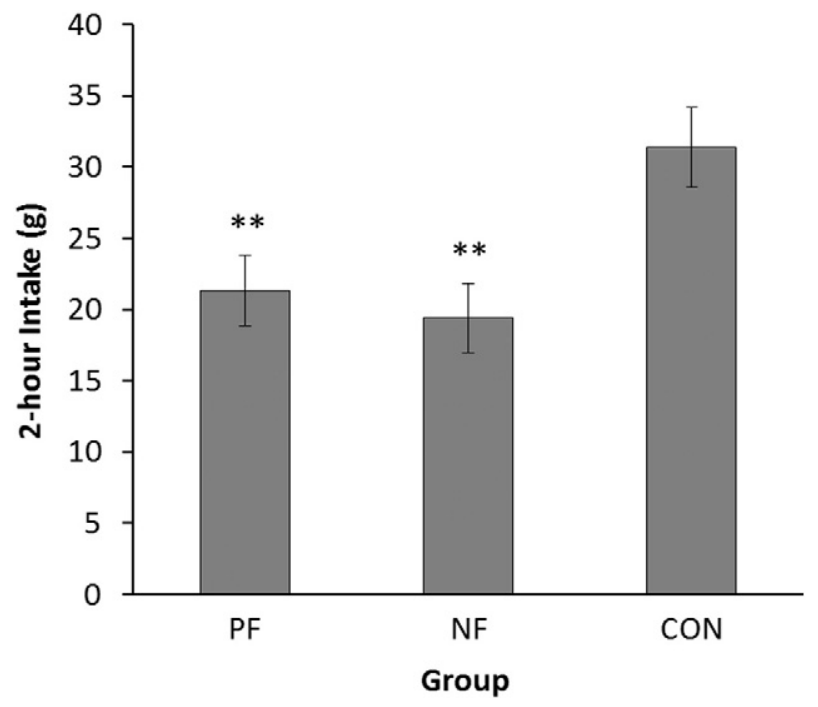

Fig. 3. Mean \pm SEM intakes of a $2 \%$ sucrose $+0.2 \%$ saccharin mixture after a period of food restriction. Asterisks indicate significant difference from $\operatorname{CON}\left({ }^{* *} p<0.01\right)$.

\subsubsection{Progressive ratio lever-pressing for sucrose reinforcers}

Despite the large differences in consumption, all groups behaved similarly in the progressive ratio task (Table 1 ). Groups did not differ in the number of sucrose pellets earned at the breakpoint, $\mathrm{F}(2,32)=$ 0.177 , n.s. There were also no significant differences in total lever presses or the time it took to reach the breakpoint (both Fs $<1.0$ ). Thus differences in ad lib sweet consumption were not correlated with differences in motivation to obtain sucrose pellets.

\subsubsection{Lick microstructure}

Several between-group differences were observed in lick patterns. Total licks, average lick burst size, and average number of bursts per session for each group at each sucrose concentration are depicted in Fig. 4.

Total licks in a session varied as a function of concentration, significant main effect, $\mathrm{F}(1.49,47.86)=18.57, p<0.001$. Total licks decreased at the highest concentration, presumably reflecting the satiating potency of $30 \%$ sucrose. The group differences in total licking (main effect of group, $\mathrm{F}(2,32)=4.805, p<0.05$ ) are consistent with the prior observations of sweet consumption, in that $\mathrm{CON}$ rats had the highest number of licks. However in this case total licks by NF rats were similar to CON, whereas PF rats licked significantly less than the other two groups (post hoc LSD, $p<0.01$ vs CON, $p<0.05$ vs NF). Pairwise $t$-tests confirm group PF licked less than CON at the $5 \%$ and $10 \%$ concentrations. There was no Concentration $\times$ Group interaction $(F(2.99,47.86)=1.10$, n.s. $)$.

The critical measurement of interest was lick burst size, which is interpreted as an indicator of hedonic value. As would be expected, average burst size increased significantly with sucrose concentration (main effect of concentration, $\mathrm{F}(2,64)=27.31, p<0.001$ ). There was not a simple difference between groups (main effect of Group on average burst size, $F(2,32)=2.72$, n.s.), but there was a significant Group $\times$ Concentration interaction $(\mathrm{F}(4,64)=2.79, p<0.05)$, because

\section{Table 1}

Mean \pm SEM values of three performance measures in the operant progressive ratio sessions. Data are the average of two sessions in which lever pressing was reinforced by delivery of $45 \mathrm{mg}$ sucrose pellets on a progressive ratio schedule. There are no statistically significant group differences on any measure.

\begin{tabular}{llll}
\hline & \multicolumn{2}{l}{ Group } & \\
\cline { 2 - 4 } & PF & NF & CON \\
\hline Reinforcers obtained & $14.1 \pm 0.59$ & $13.6 \pm 0.74$ & $13.6 \pm 0.96$ \\
Total lever presses & $503 \pm 66.5$ & $466 \pm 73.9$ & $493 \pm 97.4$ \\
Session length (min) & $56.6 \pm 1.4$ & $60.7 \pm 3.4$ & $61.7 \pm 3.2$ \\
\hline
\end{tabular}



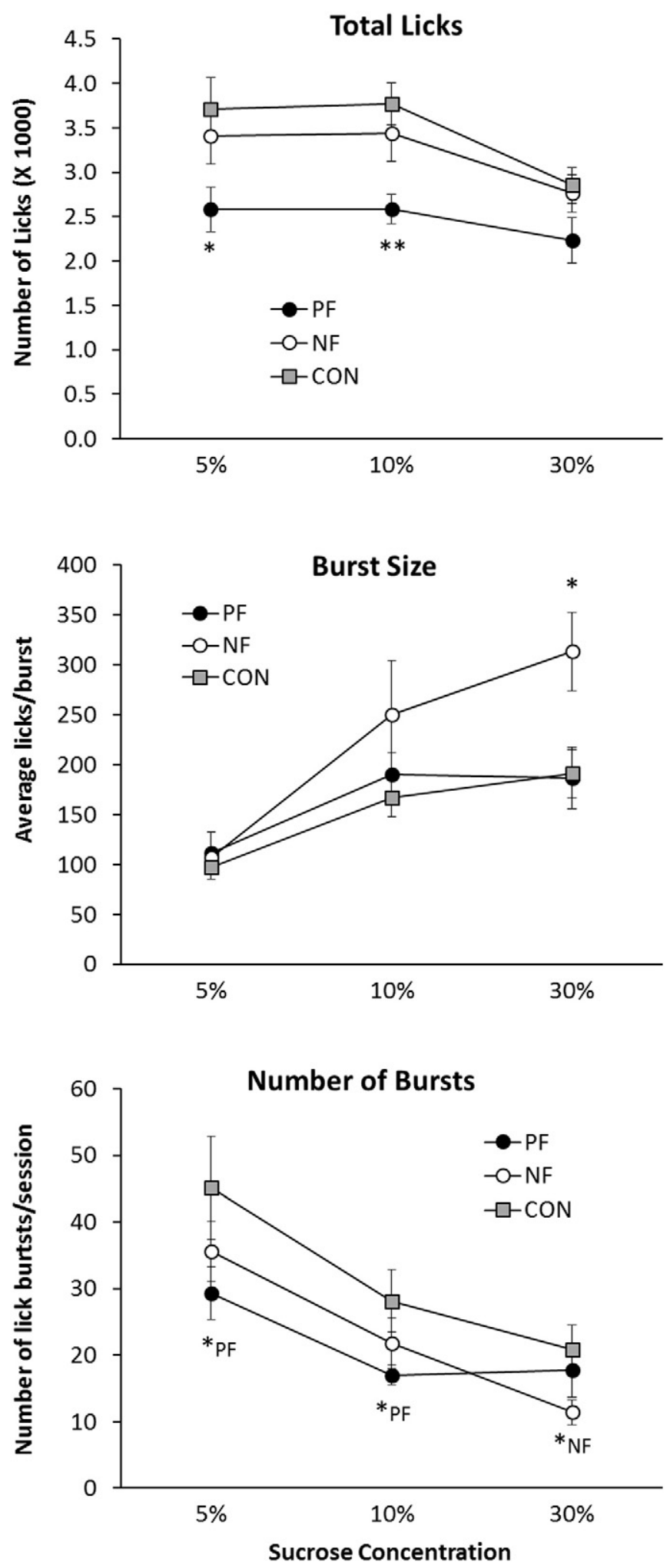

Fig. 4. Total lick counts, size of lick bursts, and lick burst counts for the series of 30-min licking microstructure sessions conducted with $5 \%, 10 \%$, and 30\% sucrose. Each rat was tested twice at each concentration in counterbalanced order and the two sessions at each concentration were averaged for analysis. Asterisks indicate significantly difference from $\mathrm{CON}$ at that concentration.

as concentration increased, burst size increased more in the NF group than it did for the other groups. Pairwise contrasts confirm NF rats had larger lick bursts than CON at the $30 \%$ concentration $(p<0.05)$.
Group NF's larger burst size at $10 \%$ concentration was not statistically significant $(p=0.06)$.

The number of lick bursts decreased as sucrose concentration increased [main effect of concentration, $\mathrm{F}(1.452,46.453)=41.771$, $p<0.001$ ], which reflects the combined influence of increasing burst size offset by the increasing satiating potency of more concentrated sucrose. There was no overall group difference on this measure (main effect of Group, $\mathrm{F}(2,32)=2.17$, n.s, Concentration $\times$ Group interaction, $\mathrm{F}(2.90,46.45)=2.02$. n.s.) though pairwise contrasts indicate group PF had lower lick burst counts than CON at the $5 \%$ and $10 \%$ concentrations, and the NF group has a lower burst count at the $30 \%$ concentration.

\subsubsection{Body weights}

At the outset of this phase, the weight differences that had accrued in Experiment 1 were no longer evident since the cafeteria diets had been withheld during the several weeks of flavor nutrient learning measurements and then operant training. When cafeteria diets resumed for three weeks preceding lick microstructure testing, weight differences reappeared (main effect of Group, $\mathrm{F}(2,33)=9.44, p<0.01$ ). Average weights (mean $\pm \mathrm{SD}$ ) for CON, PF, and NF were $321.7 \pm 30.0$, $400.0 \pm 66.1$, and $325.1 \pm 32.7$, respectively. Similar to Experiment 1, on the cafeteria diet the PF rats were heavier than the CON, $t(22)=$ $16.07, p<0.01$, and heavier than NF, $t(22)=14.56, p<0.01$ but CON and NF remained similar to one another $(p=0.79)$.

\subsection{Discussion}

In this experiment PF and NF rats once again consumed less sweet solution than CON rats, in this case a highly-preferred sucrose + saccharin mixture, replicating the effect seen previously with $6.5 \%$ glucose and with $1 \%$ glucose $+0.125 \%$ saccharin. Thus, effects of PF and NF diets on sweet consumption appear to be relatively robust and are not limited to a specific sweetness level or type of sweetener. This effect was also seen, but only for PF rats, across the range of sucrose solutions used in lick microstructure. In those tests volume consumed was not directly measured because the lick counts are a close proxy. The consumption effect was this time weak or absent for NF rats, but PF rats had significantly lower lick counts than CON.

Unfortunately, while confirming decreased sweet consumption following a history of either cafeteria diet type, this experiment does not provide a clear explanation for that decrease. It does rule out some key possibilities. First, groups did not differ in any measure of the operant progressive ratio task, thus they are likely not differently motivated to obtain sucrose nor is sucrose a less effective reinforcer. Second, lick microstructure analysis revealed group differences in sucrose palatability but they did not parallel the group differences in sweet consumption. In fact the lick microstructure result for NF is paradoxical: despite lower ad lib sweet consumption than CON in several tests, the NF rats treated high concentrations of sucrose as more palatable than the other groups. Moreover, while they consumed less than CON of several low-intensity sweets (e.g., 6.5\% glucose, the $\mathrm{G}+\mathrm{S}$ solution) their licking microstructure at $5 \%$ sucrose was identical to CON. PF rats' consistent tendency to consume less sweet solution seen in several tests was not accompanied by any apparent palatability difference from CON, according to the lick burst size measures. In sum, cafeteria diet history appears to reduce subsequent sweet intake but this is not explained by a simple motivational difference or a simple palatability difference. More work will be necessary to explain these effects, and some speculations are offered in the General Discussion.

\section{General discussion}

The impetus for this research is the apparent discrepancy between flavor-nutrient conditioning experiments in animal models and human participants. In extensive animal work, effects of flavornutrient conditioning are highly replicable and quite robust, as 
demonstrated by single-trial learning [28,37], powerful CS + preferences and increased meal sizes [10-12], avid consumption of initiallyavoided tastes $[10,38]$, shifts in hedonic taste reactivity $[39,40]$ and progressive ratio licking [41], and resistance to extinction [28,42,43]. Rats can learn to prefer one flavor over another even if it signals only modest energetic advantage, such as a flavor paired with intragastric infusion of a dilute $1 \%$ maltodextrin solution $(0.04 \mathrm{kcal} / \mathrm{g})$ versus water infusion [44]. These observations all attest to the adaptive significance and psychological primacy of this learning system.

In contrast, attempts to extend this model to humans have produced several positive findings but also several null results, and effects are sometimes weak or unreliable. While some studies have shown clear evidence of de novo learning produced under controlled conditions (e.g., [45-52]) several others report null results (e.g., [53-57]). Sometimes results are seen in only some participants (e.g., unrestrained eaters [58]) or in some dependent measures (e.g., liking, intake, choice) but not others.

This discrepancy is important for several reasons. First, for translational research to make use of animal models it will be necessary to determine if the discrepancy simply reflects procedural and measurement issues or more fundamental differences in the psychological controls of eating behavior. More broadly it calls attention to various potential ways that the modern obesogenic environment, as a radical shift from the environment of evolutionary adaptation, may disrupt or dysregulate the systems that would ordinarily orchestrate adaptive physiological and behavioral responses to food.

The main aim of the present work was to test the hypothesis that the modern, processed diet impairs flavor-nutrient learning. The average American supermarket stocks over 42,000 products [59]. Industrial food processing creates practically endless variety of different flavor and ingredient combinations, and global commerce enables access to foods from different cultures and climates that would have remained unattainable even in the fairly recent past. Even if most people consume a small subset of that enormous range, a typical consumer would still routinely experience differently flavored versions of the same food and similar flavorings in nutritionally different foods. Quite unlike our ancestral past in which taste and flavor perception were valuable guides to selecting the safest and most beneficial energy sources, in a modern, processed diet flavor is all but irrelevant to nutrient content, functioning more like entertainment.

The flavor confusion hypothesis attempts to explain unreliable findings in human flavor-nutrient experiments as a learning impairment caused by this background experience. This would mean that human experiments have difficulty producing flavor-nutrient learning because the average adult living in the industrialized environment has, in a manner of speaking, given up on learning (or, at least new learning). Yet the flavor-nutrient conditioning results of the present experiments failed to support this view. Rats maintained on a highly variable diet of processed, flavored foods were in no way impaired at flavor-nutrient conditioning compared to rats maintained on a monotonous, invariant chow diet, or compared to rats consuming a diverse but ostensibly unconfusing minimally-processed diet. Of course it could be said that the diet of 96 processed foods was insufficient to fully model the variability of the modern diet. But when conditioned acceptance was measured PF rats actually showed stronger learning instead of the predicted impairment. In acceptance tests PF rats better discriminated between $\mathrm{CS}+$ and CS- flavors by increasing their $\mathrm{CS}+$ intake. For that reason along with the effects on ad lib sweet taste acceptance, we can conclude the PF diet was sufficient to alter behavior, just not in the way predicted by the flavor confusion hypothesis.

One explanation for the more successful flavor discrimination by the PF group in the acceptance tests could be that the PF diet, instead of impairing learning, actually provided more practice at discerning flavor-nutrient relationships. Experience on a diet with more flavornutrient variability may have required more attention and required rats to represent foods' sensory attributes as complex configural stimuli rather than simple cues. In this view, the PF diet may produce flavor expertise rather than confusion. In light of this hypothesis, NF rats would have and did perform at a level intermediate between PF and CON rats, because they could have benefited from their history of variety in some sensory cues like sweetness or qualitative similarities among some foods in their diet.

The idea that sensory variability in the PF diet enabled better performance on subsequent flavor-nutrient conditioning is consistent with some work in taste aversion learning, showing that prior experience with different tastes/flavors can sometimes improve subsequent performance on a novel taste discrimination task [60-62]. A related possibility is that prior sweetness experience facilitated PF rats' ability to attend to the unique CS flavors during training, and not the sweetness the CSs shared in common. Among CON rats who had much less prior sweet exposure (only that provided in the initial acclimation sessions) the sweetness in the CS solutions may have made them more similar. Again this idea accurately predicts that learning by the NF rats would be intermediate between PF and CON. If this 'flavor expertise' effect is valid, the potential implication for flavor-nutrient conditioning in humans would be to look not at properties of the diet, but rather at situational and contextual variables that interfere with appropriate attentional focus during eating as the factor that impairs learning.

Non-support for the flavor confusion hypothesis in this experiment is not to say that the modern processed diet has no detrimental effects on the learned controls of appetite in humans and animal models. Others have demonstrated that incorporation of non-nutritive sweeteners and fat replacers in the diet alters the physiological and behavioral responses to those sensory cues in ways that can lead to overeating $[20-23,63]$, and both brain imaging studies $[64,65]$ and test meal studies [66] show differences in appetitive responses correlated with habitual use of non-nutritive sweeteners. A diet high in saturated fats and refined carbohydrates can damage brain systems responsible for modulating appetitive responses based on interoceptive state cues [67,68]. Habitual sensory-nutrient inconsistency may lead to deficits in accurately forecasting the consequences of food consumption, as dietary variability correlates with lower expected satiety from familiar foods [19]. Pervasive exposure to food cues in the environment may trigger excessive cue reactivity in sensitive individuals, eventually establishing a syndrome of craving and binge eating [69]. Thus, there are certainly many ways that the modern food environment challenges the adaptive functioning of learning systems that evolved in an environment of food scarcity. But our present findings suggest that this one notable attribute of the modern food environment - the alleged poor predictive validity of flavor cues in highly processed food - does not specifically impair learning to prefer and increased intake of flavors paired with postingestive nutrient sensing as commonly observed in animal models.

Our finding that conditioned acceptance learning was apparently superior in PF rats is especially striking given evidence of other ways that cafeteria diets may impair learning systems relevant to food preference and meal size. In particular, rats fed a cafeteria diet of palatable energy dense foods (similar to our PF diet though less varied) showed attenuated sensory specific satiety, and impaired performance in a test of outcome learning in which approach behavior should be guided by the sensory features of an expected reinforcer [26]. If we presume that the $\mathrm{PF}$ rats in the present experiment would be similarly impaired in stimulus-outcome tasks, it then follows that increased acceptance of a nutrient-predictive CS+ flavor is not fundamentally based on representing any expected outcome of consuming it. This may reflect an important distinction between mechanisms of flavor-nutrient learning as studied in animal models, and impaired anticipated satiety that follows from dietary variability in humans (e.g., [19]). But this point is speculative, as the question of outcome representation in flavornutrient learning has not been thoroughly addressed in the literature.

The PF cafeteria diet did affect body weight. After three months on the experimental diets PF rats were significantly heavier than rats in the other two groups. That observation on its own is unsurprising 
given the high palatability and higher average energy density of the PF diet. However, since this was to our knowledge the first study to include a "natural foods" group in comparison to the typical highly-processed cafeteria diet, we can conclude that dietary variety per se is not a dysregulatory influence on body weight. The NF diet was highly varied diet but lower in energy density, at least on average. (However, the NF foods were not all low energy. Approximately $20 \%$ of the NF foods were similar or higher in energy density than chow. Some NF foods, such as nuts, were just as energy dense as anything in the PF diet.)

Simple effects on bodyweight or total energy intake were unlikely to be the main source of any of behavioral differences we observed. Both cafeteria groups increased their CS + acceptance in flavor nutrientconditioning more than $\mathrm{CON}$, and both reduced their ad lib consumption of sweet solutions, even though only PF rats were heavier. NF rats responded to sucrose the most differently from $\mathrm{CON}$ in lick microstructure tests despite similarity in bodyweight.

An unexpected but robust effect in this study was that both types of cafeteria diet caused lasting reduction relative to CON in sweet solution consumption during ad lib access. This was true for the $1 \%$ glucose $+0.125 \%$ saccharin mixture and the $6.5 \%$ glucose solution used in flavor-nutrient training, and was replicated again with a sweeter $2 \%$ sucrose $+0.2 \%$ saccharin mixture. We were surprised by this effect, since habitual consumption of sweets throughout early life is generally correlated with lasting sweet preference and intake in humans [70-72].

Reduced sucrose consumption by PF rats is also surprising in light of the 'sugar addiction' model in which frequent, limited-access consumption of sucrose leads to withdrawal-like symptoms and binge consumption after abstinence [73]. The PF diet involved many sugary foods provided frequently but in limited amounts. Since cafeteria diets were then withheld during periods of behavioral testing, one may have expected stimulation of sweet consumption. But our finding is not unprecedented, as other recent studies have reported that rats maintained on a palatable high-energy maintenance diets subsequently consume less sucrose than chow-only controls [74,75]. There is also evidence that prior extended access to a palatable, high energy cafeteria-diet changes lick microstructure, suggesting decreased palatability [76]. Our present data largely fit with this prior work, but not entirely, as we found decreased ad lib intake $[74,75]$ but did not observe microstructural differences consistent with decreased palatability [76]. This latter discrepancy may simply reflect the range of sucrose concentrations we used for lick microstructure being higher than those used by [76]. It would stand to reason, as those authors suggested, perceived palatability of less-sweet foods would be reduced the most, in contrast to much sweeter foods of the cafeteria diet.

While these prior results coincide with the PF group in our study, whose diet was palatable, energy dense, and promoted excess weight gain, it is unlikely to also account for the NF rats, who were not overweight and whose diet was not high in simple sugars or fat. Since we are unaware of any prior studies using a diet like our NF diet, it is difficult to say what attribute of the NF rats' experience affected their sweet taste acceptance. Experiment 2 was undertaken to explore this effect further.

The effect of cafeteria diet history on sweet intake was not obviously correlated with altered reinforcing value of sugar as measured by the progressive ratio operant task. One caveat is that by the time of the progressive measurements, the effect of PF diet on bodyweight dissipated and PF rats' weights were similar to the other groups. Other studies have found effects of chronic excess energy consumption and dietinduced obesity on progressive ratio tasks, but studies have found opposite effects, including both increased and decreased PR breakpoints [31, 77]. The degree of obesity, the length of time on the high-energy diet, and its sensory properties may all play roles in shifting motivation [31]. Thus more work on this issue would be worthwhile, as the effects of prior diet history and overweight on progressive ratio tasks appears complex. In any case, our rationale for including progressive ratio tasks in this experiment was to seek potential explanations for group differences in ad lib consumption, which notably were independent of body weight.

Since all groups in the present study behaved similarly in the progressive ratio task, we can conclude that mere experience with sweet foods or with highly palatable foods or with dietary diversity is not on its own a source of the motivational shifts than have been seen in diet induced obesity. However this conclusion is limited, first, in that all the group differences in consumption involved sweet solutions, whereas the operant task was reinforced with solid sucrose pellets. It is unknown whether that may have obscured potential differences that may have been observed if using a reinforcer similar in concentration/ intensity to the ad lib intake tests. Again, additional work assessing motivational impact of a range of sweet intensities following these cafeteria diet histories will be useful.

To examine whether diet history altered the hedonic impact of sweet solutions we examined lick microstructure across a range of sucrose concentrations. The key parameter of interest was lick burst size. By this measure NF rats treated higher concentrations of sucrose as significantly more palatable than did PF or CON rats. This is paradoxical, as it is unclear why greater perceived palatability would explain lower intake of sweets. The large difference in burst size between NF rats and CON rats at the $30 \%$ concentration raises questions about why experience on a natural foods diet makes a high concentration of sucrose so palatable. One possibility is that NF rats experienced a positive contrast effect [78]. NF rats' exposure to simple sugars would have been in fruits and vegetables, which also contain unattractive sour, bitter, and astringent compounds. When subsequently tested with pure sugar solutions a positive contrast effect could have increased the sugar's hedonic impact. This is consistent with the idea that the actual functional significance of sweet taste liking for foraging animals is not as a "cue for calories" (fruits and vegetables are really quite low in energy density, and starchy foods are a better carbohydrate source) but rather to render palatable the otherwise aversive sources of essential micronutrients.

In sum, the results of ad lib sweet consumption tests and lick microstructure are enigmatic. The cafeteria diets significantly decreased ad lib sweet intake in both NF and PF rats, despite PF rats showing no differences from CON in lick microstructure and NF rats exhibiting paradoxical increased sugar palatability, and neither group differed in progressive ratio responding. Ultimately we can conclude that neither simple changes in reinforcing value nor palatability explain the effects of cafeteria diets on sweet intake, nor are these effects secondary to weight gain. There are clearly additional behavioral mechanisms involved in the evaluation of sweet taste that will need to be explored, as it is likely sweet consumption was suppressed for different reasons in PF and NF rats.

Returning to the original rationale for this experiment, our results serve primarily to cast some doubt on the idea that sensory variability of the modern diet is a disruptive factor in flavor-nutrient learning. Other explanations should be systematically pursued to explain the discrepancies between animal and human studies. It may be that subtle design and measurement differences play some role, but it will also be informative to determine the extent to which various higher-order cognitive, social and cultural influences constrain or override more basic learning and reward systems.

An alternative formulation of the flavor confusion hypothesis may still explain the chief difficulty with human studies: perhaps experiments have difficulty producing de novo learning, not because dietary variety impairs the learning of novel flavor-nutrient relationships, but rather because the modern diet makes it difficult for experimenters to provide truly novel relationships. That is, to what extent are the stimuli used in human experiments actually just relatively minor variations on participants' extensive previous experiences? CS novelty is well known to influence basic conditioning processes and there is evidence that CS novelty is characteristic of successful flavor-nutrient experiments in humans [18]. The capacity of humans for top-down processing based on cognitive schemata may mean that even distinctively flavored food 
stimuli given in experiments (e.g., soups, yogurts, drinks) are experienced as instances of a highly familiar, over-learned category, and are processed fundamentally differently than truly novel stimuli.

Thus two fundamentally different views of this dilemma emerge. One proposes that flavor-nutrient conditioning is difficult to produce in human experiments because the learning system functions poorly. (This could mean either it is a relatively unimportant learning ability for humans compared to social and cultural influence, or that it is impaired by the environment.) The other is that humans have an extensive capacity for flavor-nutrient learning and that it strongly influences appetite, but each individual already has such rich and extensive experience to draw on that by adulthood it is rare encounter something new to learn about. Ultimately both views would mean that new learning is rare but they make opposite claims about the status of flavor-nutrient associations in shaping motivation and food intake. It has recently been proposed that cross-cultural research can be valuable for these questions [79]. We also conclude from our present results that animal models can be improved by incorporating key features of the modern obesogenic environment and diet to better understand the roles that flavor-nutrient conditioning plays in environmentally-induced overeating.

\section{Acknowledgements}

The rationale for this research originally emerged from conversations with Dr. Jeffrey Brunstrom. We are grateful for his thoughtful input on connections between animal and human research on dietary learning. A thesis based on this project was previously submitted to the faculty of Bucknell University by K.M.P. for the Bachelor's degree with Honors. Drs. Jennie Stevenson and Mizuki Takahashi provided helpful feedback at that stage. Meredith Lutz and Julia Mammone provided valuable technical assistance. We are grateful to Drs. Alan Spector and Lindsey Schier for helpful comments on a previous draft of the manuscript. The research was supported by a Bucknell University Dean's Fellowship to K.P.M.

\section{References}

[1] A. Sclafani, K. Ackroff, The role of gut nutrient sensing in stimulating appetite and conditioning food preferences, Am. J. Physiol. Regul. Integr. Comp. Physiol. 302 (2012) R1119-R1133.

[2] K.P. Myers, Why we eat what we eat, in: N.M. Avena (Ed.), Hedonic Eating: How the Pleasurable Aspects of Food Can Affect Our Brains and Behavior, Oxford University Press 2015, pp. p9-38.

[3] K.P. Myers, A. Sclafani, Development of learned flavor preferences, Dev. Psychobiol. 48 (2006) 380-388

[4] M.R. Yeomans, The role of learning in development of food preferences, Frontiers Nutr. Sci. 3 (2006) 93.

[5] A. Sclafani, Oral and postoral determinants of food reward, Physiol. Behav. 81 (2004) 773-779.

[6] A. Sclafani, Macronutrient-conditioned flavor preferences, in: H. Berthoud, R. Seeley (Eds.), Neural and Metabolic Control of Macronutrient Intake, CRC Press, Boca Raton, FL 1999, pp. p93-107.

[7] E.A. Burritt, F.D. Provenza, Lambs form preferences for nonnutritive flavors paired with glucose, J. Anim. Sci. 70 (1992) 1133-1136.

[8] F.D. Provenza, Postingestive feedback as an elementary determinant of food preference and intake in ruminants, Journal of Range Management Archives 48 (1995) $2-17$.

[9] J. Figueroa, D. Solà-Oriol, E. Borda, A. Sclafani, J.F. Pérez, Flavour preferences conditioned by protein solutions in post-weaning pigs, Physiol. Behav. 107 (2012) 309-316.

[10] C. Perez, F. Lucas, A. Sclafani, Increased flavor acceptance and preference conditioned by the postingestive actions of glucose, Physiol. Behav. 64 (1998) 483-492.

[11] K.P. Myers, A. Sclafani, Conditioned enhancement of flavor evaluation reinforced by intragastric glucose: I. Intake acceptance and preference analysis, Physiol. Behav. 74 (2001) 481-493.

[12] G. Elizalde, A. Sclafani, Flavor preferences conditioned by intragastric polycose infusions: a detailed analysis using an electronic esophagus preparation, Physiol. Behav. 47 (1990) 63-77.

[13] K. Ackroff, A. Sclafani, Energy density and macronutrient composition determine flavor preference conditioned by intragastric infusions of mixed diets, Physiol. Behav. 89 (2006) 250-260.

[14] K. Ackroff, Learned flavor preferences. The variable potency of post-oral nutrient reinforcers, Appetite 51 (2008) 743-746.
[15] C. Perez, F. Lucas, A. Sclafani, Carbohydrate, fat, and protein condition similar flavor preferences in rats using an oral-delay procedure, Physiol. Behav. 57 (1995) 549-554

[16] F. Lucas, A. Sclafani, Flavor preferences conditioned by intragastric fat infusions in rats, Physiol. Behav. 46 (1989) 403-412.

[17] J.M. Brunstrom, Dietary learning in humans: Directions for future research, Physiol. Behav. 85 (2005) 57-65.

[18] M.R. Yeomans, Flavour-nutrient learning in humans: an elusive phenomenon? Physiol. Behav. 106 (2012) 345-355.

[19] C.A. Hardman, D. Ferriday, L. Kyle, P.J. Rogers, J.M. Brunstrom, So many brands and varieties to choose from: does this compromise the control of food intake in humans? PLoS One 10 (2015) e0125869.

[20] T.L. Davidson, S.E. Swithers, A Pavlovian approach to the problem of obesity, Int. J. Obes. 28 (2004) 933-935.

[21] S.E. Swithers, T.L. Davidson, Influence of early dietary experience on energy regulation in rats, Physiol. Behav. 86 (2005) 669-680.

22] S.E. Swithers, A.A. Martin, T.L. Davidson, High-intensity sweeteners and energy balance, Physiol. Behav. 100 (2010) 55-62.

[23] S.E. Swithers, A.F. Laboy, K. Clark, S. Cooper, T. Davidson, Experience with the highintensity sweetener saccharin impairs glucose homeostasis and GLP-1 release in rats, Behav. Brain Res. 233 (2012) 1-14.

[24] Z.S. Warwick, S.S. Schiffman, Flavor-calorie relationships: effect on weight gain in rats, Physiol. Behav. 50 (1991) 465-470.

[25] C. Pérez, L. Fanizza, A. Sclafani, Flavor preferences conditioned by intragastric nutrient infusions in rats fed chow or a cafeteria diet, Appetite 32 (1999) 155-170.

[26] A.C. Reichelt, M.J. Morris, R. Westbrook, Cafeteria diet impairs expression of sensoryspecific satiety and stimulus-outcome learning, Front. Psychol. 5 (2014).

[27] Z.S. Warwick, H.P. Weingarten, Dissociation of palatability and calorie effects in learned flavor preferences, Physiol. Behav. 55 (1994) 501-504.

[28] K.P. Myers, Robust preference for a flavor paired with intragastric glucose acquired in a single trial, Appetite 48 (2007) 123-127.

[29] S. Reilly, Reinforcement value of gustatory stimuli determined by progressive ratio performance, Pharmacol. Biochem. Behav. 63 (1999) 301-311.

[30] A. Sclafani, K. Ackroff, Reinforcement value of sucrose measured by progressive ratio operant licking in the rat, Physiol. Behav. 79 (2003) 663-670.

[31] A.L. Tracy, C.J. Wee, G.E. Hazeltine, R.A. Carter, Characterization of attenuated food motivation in high-fat diet-induced obesity: critical roles for time on diet and reinforcer familiarity, Physiol. Behav. 141 (2015) 69-77.

[32] K. Brennan, D. Roberts, H. Anisman, Z. Merali, Individual differences in sucrose consumption in the rat: motivational and neurochemical correlates of hedonia, Psychopharmacology 157 (2001) 269-276.

[33] J.D. Davis, G.P. Smith, Analysis of the microstructure of the rhythmic tongue movements of rats ingesting maltose and sucrose solutions, Behav. Neurosci. 106 (1992) 217.

[34] J.D. Davis, G.P. Smith, Analysis of lick rate measure the positive and negative feedback effects of carbohydrates on eating, Appetite 11 (1988) 229-238.

[35] D.M. Dwyer, Licking and liking: the assessment of hedonic responses in rodents, Q. J. Exp. Psychol. 65 (2012) 371-394.

[36] N.R. Richardson, D.C. Roberts, Progressive ratio schedules in drug selfadministration studies in rats: a method to evaluate reinforcing efficacy, J. Neurosci. Methods 66 (1996) 1-11.

[37] K. Ackroff, C. Dym, Y.M. Yiin, A. Sclafani, Rapid acquisition of conditioned flavor preferences in rats, Physiol. Behav. 97 (2009) 406-413.

[38] K.P. Myers, A. Sclafani, Conditioned acceptance and preference but not altered taste reactivity responses to bitter and sour flavors paired with intragastric glucose infusion, Physiol. Behav. 78 (2003) 173-183.

[39] C.A. Forestell, V.M. LoLordo, Palatability shifts in taste and flavour preference conditioning O. J. Exp. Psychol. B 56 (2003) 140-160

[40] K.P. Myers, A. Sclafani, Conditioned enhancement of flavor evaluation reinforced by intragastric glucose. II. Taste reactivity analysis, Physiol. Behav. 74 (2001) 495-505.

[41] A. Sclafani, K. Ackroff, Nutrient-conditioned flavor preference and incentive value measured by progressive ratio licking in rats, Physiol. Behav. 88 (2006) 88-94.

[42] D.B. Drucker, K. Ackroff, A. Sclafani, Nutrient-conditioned flavor preference and acceptance in rats: effects of deprivation state and nonreinforcement, Physiol. Behav. 56 (1994) 701-707.

[43] A. Sclafani, J.W. Nissenbaum, Robust conditioned flavor preference produced by intragastric starch infusions in rats, Am. J. Phys. 255 (1988) R672-R675

[44] K. Ackroff, A. Sclafani, Flavor preferences conditioned by intragastric infusions of dilute polycose solutions, Physiol. Behav. 55 (1994) 957-962.

[45] K. Appleton, R. Gentry, R. Shepherd, Evidence of a role for conditioning in the development of liking for flavours in humans in everyday life, Physiol. Behav. 87 (2006) 478-486.

[46] L.L. Birch, L. McPhee, L. Steinberg, S. Sullivan, Conditioned flavor preferences in young children, Physiol. Behav. 47 (1990) 501-505.

[47] D.L. Kern, L. McPhee, J. Fisher, S. Johnson, L.L. Birch, The postingestive consequences of fat condition preferences for flavors associated with high dietary fat, Physiol. Behav. 54 (1993) 71-76.

[48] S. Johnson, L. McPhee, L. Birch, Conditioned preferences: young children prefer flavors associated with high dietary fat, Physiol. Behav. 50 (1991) 1245-1251.

[49] E.D. Capaldi, G.J. Privitera, Flavor-nutrient learning independent of flavor-taste learning with college students, Appetite 49 (2007) 712-715.

[50] S. Mobini, L.C. Chambers, M.R. Yeomans, Effects of hunger state on flavour pleasantness conditioning at home: Flavour-nutrient learning vs. flavour-flavour learning, Appetite 48 (2007) 20-28.

[51] M.R. Yeomans, M. Leitch, N.J. Gould, S. Mobini, Differential hedonic, sensory and behavioral changes associated with flavor-nutrient and flavor-flavor learning, Physiol. Behav. 93 (2008) 798-806. 
[52] E. Remy, C. Divert, J. Rousselot, L. Brondel, S. Issanchou, S. Nicklaus, Impact of energy density on liking for sweet beverages and caloric-adjustment conditioning in children, Am. J. Clin. Nutr. 100 (2014) 1052-1058.

[53] D. Mela, F. Trunck, J. Aaron, No effect of extended home use on liking for sensory characteristics of reduced-fat foods, Appetite 21 (1993) 117-129.

[54] S. Specter, F. Bellisle, S. Hémery-Véron, P. Fiquet, F. Bornet, G. Slama, Reducing ice cream energy density does not condition decreased acceptance or engender compensation following repeated exposure, Eur. J. Clin. Nutr. 52 (1998) 703-710.

[55] S.J. Caton, S.M. Ahern, E. Remy, S. Nicklaus, P. Blundell, M.M. Hetherington, Repetition counts: Repeated exposure increases intake of a novel vegetable in UK preschool children compared to flavour-flavour and flavour-nutrient learning, Br. J. Nutr. 109 (2013) 2089-2097.

[56] G.G. Zeinstra, M. Koelen, F. Kok, C. De Graaf, Children's hard-wired aversion to pure vegetable tastes. A 'failed' flavour-nutrient learning study, Appetite 52 (2009) 528-530.

[57] E. Zandstra, K. Stubenitsky, C. De Graaf, D. Mela, Effects of learned flavour cues on short-term regulation of food intake in a realistic setting, Physiol. Behav. 75 (2002) 83-90.

[58] J.M. Brunstrom, G.L. Mitchell, Flavor-nutrient learning in restrained and unrestrained eaters, Physiol. Behav. 90 (2007) 133-141.

[59] Food Marketing Institute. Supermarket facts. <http://www.fmi.org/research-resources/supermarket-facts $>$. Accessed 2015, 22 September.

[60] R.C. Honey, G. Hall, Enhanced discriminability and reduced associability following flavor preexposure, Learn. Motiv. 20 (1989) 262-277.

[61] V. Scahill, N. Mackintosh, The easy to hard effect and perceptual learning in flavor aversion conditioning, J. Exp. Psychol. Anim. Behav. Processes 30 (2004) 96.

[62] M. Symonds, G. Hall, Stimulus preexposure, comparison, and changes in the associability of common stimulus features, The Quarterly Journal of Experimental Psychology: Section B 50 (1997) 317-331.

[63] S. Swithers, A. Doerflinger, T. Davidson, Consistent relationships between sensory properties of savory snack foods and calories influence food intake in rats, Int. J. Obes. 30 (2006) 1685-1692.

[64] E. Green, C. Murphy, Altered processing of sweet taste in the brain of diet soda drinkers, Physiol. Behav. 107 (2012) 560-567.

[65] K. Rudenga, D. Small, Amygdala response to sucrose consumption is inversely related to artificial sweetener use, Appetite 58 (2012) 504-507.
[66] K. Appleton, P. Rogers, J. Blundell, Effects of a sweet and a nonsweet lunch on shortterm appetite: differences in female high and low consumers of sweet/low-energy beverages, J. Hum. Nutr. Diet. 17 (2004) 425-434.

[67] S.E. Kanoski, T.L. Davidson, Western diet consumption and cognitive impairment: links to hippocampal dysfunction and obesity, Physiol. Behav. 103 (2011) 59-68.

[68] C.H. Sample, A.A. Martin, S. Jones, S.L. Hargrave, T.L. Davidson, Western-style diet impairs stimulus control by food deprivation state cues: implications for obesogenic environments, Appetite (2015).

[69] A. Jansen, A learning model of binge eating: cue reactivity and cue exposure, Behav. Res. Ther. 36 (1998) 257-272.

[70] D.G. Liem, C. De Graaf, Sweet and sour preferences in young children and adults: role of repeated exposure, Physiol. Behav. 83 (2004) 421-429.

[71] S. Park, L. Pan, B. Sherry, R. Li, The association of sugar-sweetened beverage intake during infancy with sugar-sweetened beverage intake at 6 years of age, Pediatrics 134 (Suppl. 1) (2014) S56-S62.

[72] M.Y. Pepino, J.A. Mennella, Factors contributing to individual differences in sucrose preference, Chem. Senses 30 (Suppl. 1) (2005) i319-i320.

[73] N.M. Avena, K.A. Long, B.G. Hoebel, Sugar-dependent rats show enhanced responding for sugar after abstinence: evidence of a sugar deprivation effect, Physiol. Behav. 84 (2005) 359-362.

[74] F.A. Duca, T.D. Swartz, M. Covasa, Effect of diet on preference and intake of sucrose in obese prone and resistant rats, PLoS ONE 9 (2014), e111232.

[75] Y. Treesukosol, N. Liang, T.H. Moran, Alterations in sucrose sham-feeding intake as a function of diet-exposure in rats maintained on calorically dense diets, Appetite 92 (2015) 278-286.

[76] S.I. Martire, R.F. Westbrook, M.J. Morris, Effects of long-term cycling between palatable cafeteria diet and regular chow on intake, eating patterns, and response to saccharin and sucrose, Physiol. Behav. 139 (2015) 80-88.

[77] S. La Fleur, L. Vanderschuren, M. Luijendijk, B. Kloeze, B. Tiesjema, R. Adan, A reciprocal interaction between food-motivated behavior and diet-induced obesity, Int. J. Obes. 31 (2007) 1286-1294.

[78] C.F. Flaherty, J. Largen, Within-subjects positive and negative contrast effects in rats, J. Comp. Physiol. Psychol. 88 (1975) 653.

[79] J.M. Brunstrom, P.J. Rogers, K.P. Myers, J.D. Holtzman, In search of flavour-nutrient learning. A study of the samburu pastoralists of north-central Kenya, Appetite 91 (2015) 415-425 